\title{
A two-stage method for the capacitated multi-facility location-allocation problem
}

\author{
Martino Luis*
}

College of Engineering, Mathematics and Physical Sciences,

University of Exeter,

Exeter EX4, 4QF, UK

Email: M.Luis@exeter.ac.uk

*Corresponding author

\section{Chandra A. Irawan}

Nottingham University Business School China, University of Nottingham Ningbo China, 199 Taikang East Road, Ningbo 315100, China

Email: Chandra.Irawan@nottingham.edu.cn

\author{
Arif Imran \\ Department of Industrial Engineering, \\ Institut Teknologi Nasional, \\ Bandung 40124, Indonesia \\ and \\ School of Industrial Engineering, \\ Telkom University, \\ Bandung 40257, Indonesia \\ Email: arifimr@yahoo.com
}

\begin{abstract}
This paper examines the capacitated planar multi-facility location-allocation problem, where the number of facilities to be located is specified and each of which has a capacity constraint. A two-stage method is put forward to deal with the problem where in the first stage a technique that discretises continuous space into discrete cells is used to generate a relatively good initial facility configurations. In stage 2 , a variable neighbourhood search (VNS) is implemented to improve the quality of solution obtained by the previous stage. The performance of the proposed method is evaluated using benchmark datasets from the literature. The numerical experiments show that the proposed method yields competitive results when compared to the best known results from the literature. In addition, some future research avenues are also suggested.
\end{abstract}

Keywords: capacitated; continuous location problem; heuristics; variable neighbourhood search; VNS.

Reference to this paper should be made as follows: Luis, M., Irawan, C.A. and Imran, A. (xxxx) 'A two-stage method for the capacitated multi-facility location-allocation problem', Int. J. Operational Research, Vol. X, No. Y, pp.000-000.

Copyright (C) 20XX Inderscience Enterprises Ltd. 
Biographical notes: Martino Luis is a Lecturer in Engineering Management at the College of Engineering, Mathematics and Physical Sciences, University of Exeter, UK. Prior to joining the University of Exeter, he was a faculty member at the Othman Yeop Abdullah Graduate School of Business, Universiti Utara Malaysia. His main research interest is in designing efficient algorithms to tackle optimisation problems such as logistics problems, facility location problems, sequencing and scheduling problems.

Chandra A. Irawan is an Assistant Professor in Operations Management at the Faculty of Business at UNNC. Prior to the University of Nottingham Ningbo China, he was a Research Fellow at the Department of Mathematics at the University of Portsmouth UK for three years involved in the leanwind project (EU 7th Framework Programme project). The project aims to reduce the cost of installation, operation, and maintenance of offshore wind farms. He was developing mathematical models and solution methods to optimise supply chain and maintenance scheduling problems in offshore wind farms. During his $\mathrm{PhD}$ at the Kent Business School at the University of Kent, he was also working as a teaching assistant.

Arif Imran is a Senior Lecturer at the Industrial Engineering, National Institute of Technology, Bandung, Indonesia. He obtained his BSc in Mathematics at the Bandung Institute of Technology, Indonesia, MSc in Industrial Engineering at the Bandung Institute of Technology and $\mathrm{PhD}$ in Operational Research at the University of Kent, England, UK. His research interests are in distribution management, heuristics optimisation and production management. His papers have appeared in EJOR, COR and IJLSM.

\section{Introduction}

In this study, we investigate the planar multi-facility location-allocation problem, known as the multi-source Weber problem (MSWP). In this problem, we are provided with a set of customers, located at $n$ fixed points, with their respective demands. We need to locate $M$ facilities in continuous space to serve these $n$ customers, and to find the allocation of these customers to these $M$ facilities in order to minimise the sum of the weighted total Euclidean distances to serve these customers. If the facilities are assumed to have unlimited capacities, the customers are served by the nearest facility (for examples, see Brimberg et al., 2008. 2014). However, in practice, this may not be true as facilities have capacity restrictions and as a result some customers may have to be served by a farther facility as the nearest facility cannot satisfy their demands. This study focuses on the MSWP in the presents of capacity constraints. This problem is referred to as capacitated multi-source Weber problem (CMSWP).

The earliest work on the CMSWP was conducted by Cooper (1972) who designed the well-known alternating transportation-location (ATL) heuristic. The ATL is an enhancement of the alternate location-allocation (ALA) method initially proposed by Cooper (1964) to solve the classical location-allocation problem. The basic idea of ATL is that the location-allocation problem and the transportation problem (TP) are alternately applied until no epsilon $(\varepsilon)$ improvement in total cost is found. Sherali and Shetty (1977) developed a convergent cutting plane algorithm to deal with the rectilinear distance CMSWP. 
In 1992, the CMSWP was restudied by Sherali and Tuncbilek (1992). They proposed a branch and bound algorithm to compute strong upper bounds via a Lagrangean relaxation scheme and a partitioning approach. Sherali et al. (1994) put forward a reformulation-linearisation technique (RLT) to solve the rectilinear distance CMSWP and reformulated the problem as a linear mixed-integer programming. Sherali et al. (2002) investigated the capacitated Euclidean and $\ell_{p}$ distances MSWP by creating a branch and bound based on a partitioning of the allocation space to construct global optimisation procedures. Zainuddin and Salhi (2007) tackled the CMSWP by designing a perturbation-based heuristic, which considered borderline customers whose locations were situated approximately half-way between their nearest and their second nearest facilities.

Aras et al. (2007a) put forward three heuristic methods which adopted Lagrangean heuristic, the $p$-median method of Hansen et al. (1998), and the cellular heuristic of Gamal and Salhi (2003) to solve the CMSWP with Euclidean, squared Euclidean, and $\ell_{p}$ distances. Aras et al. (2007b) applied simulated annealing, threshold accepting, and genetic algorithms to tackle the CMSWP with rectilinear, Euclidean, squared Euclidean, and $\ell_{p}$ distances. In a subsequent research, Aras et al. (2008) used their earlier approaches to tackle the CMSWP with rectilinear distance.

Luis et al. (2009) studied the CMSWP by proposing the concept of region-rejection which restricted some locations to be sited too close to the previously chosen locations. A discretisation technique of converting a plane into a discrete space was also put forward. Mohammadi et al. (2010) developed genetic algorithms to solve the CMSWP. Luis et al. (2011) designed a novel guided reactive greedy randomised adaptive search which integrated adaptive learning with the concept of region-rejection. Akyüz et al. (2014) put forward two forms of branch and bound algorithms where the first is based on the allocation space whereas the second on the partition of the location space to tackle the CMSWP.

Other variants on the Weber problems includes the works of Jamalian and Salahi (2014) who studied the uncapacitated multi-facility Weber problem with uncertain location of demand points and Euclidean and $\ell_{p}$ distances for both interval and ellipsoidal uncertainty sets. Fernandes et al. (2014) designed a global optimisation algorithm to solve the single facility Weber problem with limited distances and in the presences of minimum and maximum numbers of customers served. Uno et al. (2015) reformulated the Weber problem by considering random and fuzzy demands, known as the extension of the weighted Weber problems. Drezner (2015) modified the well-known Weizsfeld algorithm based on a parabolic approximation and optimality test of demand points to deal with the single facility Weber problem. Farham et al. (2015) suggested a hybrid evolutionary algorithm to solve the single facility Weber problem in the presence of congested regions where locating a facility is restricted but travelling is allowed. Hosseininezhad et al. (2015) applied a cross entropy metaheuristic to tackle the CMSWP by introducing production and installation costs.

Brimberg et al. $(2008,2014)$ provide a comprehensive review on the MSWP and in their works; a variable neighbourhood search (VNS) was also pointed out to be applied to solve efficiently the uncapacitated case. To the best of our knowledge, this is the first time that the VNS is adapted to tackle this hard variant of the Weber problem with capacity constraints. 
The remainder of this paper is structured as follows: in the next section, the mathematical formulations of the CMSWP are presented. The section thereafter discusses the proposed two-stage method. Then, a section on computational results is reported. The last section provides some conclusions and highlights some potential future research directions.

\section{The CMSWP formulations}

The CMSWP is defined as there are a set of customers, located at $n$ fixed points, with their respective demands. The aim is to locate $M$ facilities in a continuous space with their capacities $b$, and to find the allocation of these $n$ customers to these $M$ facilities without violating the capacity of any of the facilities while minimising the total sum of transportation costs. First, we present the notations and the decision variables then followed by the mathematical formulation for the CMSWP.

- Notations:

$M \quad$ the number of facilities to be located

$x_{i j} \quad$ the allocation quantities sent from facility $i$ to customer $j$,

$i=1, \ldots M$;

$j=1, \ldots, n$

$d\left(X_{i}, a_{j}\right) \quad$ is the Euclidean distance between facility $i$ and customer $j$

$a_{j}=\left(a_{j}^{1}, a_{j}^{2}\right) \in \mathfrak{R}^{2} \quad$ is the location of customer $j$

$w_{j} \quad$ the demand, or weight, of customer $j$, where $j=1, \ldots, n$

$b_{i} \quad$ a capacity of facility $i$, where $b \in N$

$N \quad$ a set of facilities' capacities.

- Decision variables

$X_{i}=\left(X_{i}^{1}, X_{i}^{2}\right) \in \Re^{2}$ are coordinates of facility $i$.

The CMSWP can be mathematically formulated as:

Minimise $\sum_{i=1}^{M} \sum_{j=1}^{n} x_{i j} d\left(X_{i}, a_{j}\right)$

Subject to

$$
\begin{aligned}
& \sum_{i=1}^{M} x_{i j}=w_{j}, \quad j=1, \ldots, n \\
& \sum_{j=1}^{n} x_{i j} \leq b_{i}, \quad i=1, \ldots M \\
& x_{i j} \geq 0, \quad i=1, \ldots M, j=1, \ldots, n
\end{aligned}
$$

The objective function (1) is to minimise the sum of the transportation costs using Euclidean distances. Constraint (2) ensures that the total demand of each customer is met. 
Constraint (3) guarantees that capacity constraints of the facilities are not violated. Constraint (4) is non-negativity constraints, which imply that the decision variables $x_{i j}$ are positive flows. It is observed that once the $M$ facilities are located, the problem becomes the generalised assignment problem. In addition, if the size of the located facilities is known, the problem reduces to the classical TP. The problem is considered to have at least one feasible solution by assuming that $\sum_{j=1}^{n} w_{j} \leq \sum_{i=1}^{M} b_{i}$.

\section{Solution method}

In this work, a two-stage method is introduced where the first stage aims to generate a relatively good solution whereas in the second stage, a metaheuristics technique is implemented to improve the solution. In the first stage, a technique called the Basic Cell Approach (BCA) is used to generate a relatively good initial facility configuration. This approach is adapted from Irawan and Salhi (2015), which was originally designed by Gamal and Salhi (2003) to solve the MSWP and it was also modified by Luis et al. (2009) to tackle the CMSWP. The BCA discretises continuous space into discrete cells based on the location of customers. This approach determines the promising cells (areas) that many customers are located. Then, initial facility locations are generated pseudo randomly within these cells and a local search based on the well-known on Cooper's ATL heuristic (Cooper, 1972) is applied to improve the facility locations.

In stage 2, a powerful metaheuristics called VNS is implemented to improve the quality of solution obtained by stage 1. In this method, Cooper's ATL is also incorporated as its local search. VNS was formally designed and proposed by Brimberg and Mladenović (1996) and Hansen and Mladenović (1997) to deal with facility location problems. VNS has been widely used to solve various hard combinatorial optimisation problems including travelling salesman problems, vehicle routing problems, location problems, scheduling, and time tabling problems, among others. A comprehensive review on variants and applications of VNS is provided by Hansen and Mladenović (2001), Hansen et al. (2010) and Brimberg et al. (2014). Figure 1 presents the main steps of the proposed two-stage method to tackle the CMSWP.

Figure 1 The main steps of the proposed two-stage method to solve the CMSWP

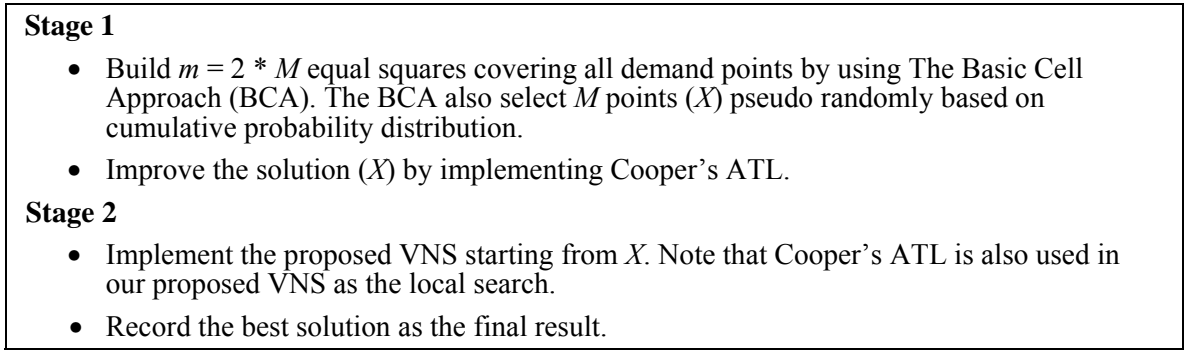

In the following subsections, the well-known Cooper's ATL is first briefly presented as this method is used in the proposed method. This is followed by a subsection explaining the main steps of the BCA and the proposed VNS algorithm. 


\subsection{Cooper's ATL heuristic}

The reasoning behind the ATL is to construct $M$ open facilities which are randomly selected from the customer locations, then the TP, using these $M$ open facilities, is applied to find the allocation for this capacitated problem. At this point, the output is the $M$ independent set of allocations, each subset consisting of $n_{i}$ customer locations where $i=1,2, \ldots, M$ and $\sum_{i=1}^{M} n_{i} \geq n$. It is worth noting that we used ' $\geq$ ' instead of '=' as some customers may have their demand split between different facilities during the allocation stage. The Weiszfeld equations, see (5), is iteratively carried out to get the new location of these $M$ facilities $(i=1,2, \ldots, M)$.

Note that the demand of some customers may have been split as a result of the solution of the TP, such as $w_{j_{i}} \leq w_{j}$. It can also be observed that some customers may be utilised more than once in (5) but every time, it is only a portion $w_{j_{i}}$ of their demand utilised. The location-allocation problem and the TP are alternately applied until no improvement in total cost can be obtained. The step by step of Cooper's ATL heuristic is illustrated in Figure 2.

$$
X_{i}^{1^{(k)}}=\frac{\sum_{j_{i}=1}^{n_{i}} \frac{w_{j_{i}} a_{j_{i}}^{1}}{d\left(X_{i}^{(k-1)}, a_{j_{i}}\right)}}{\sum_{j_{i}=1}^{n_{i}} \frac{w_{j_{i}}}{d\left(X_{i}^{(k-1)}, a_{j_{i}}\right)}} \text { and } X_{i}^{2^{(k)}}=\frac{\sum_{j_{i}=1}^{n_{i}} \frac{w_{j_{i}} a_{j_{i}}^{2}}{d\left(X_{i}^{(k-1)}, a_{j_{i}}\right)}}{\sum_{j_{i}=1}^{n_{i}} \frac{w_{j_{i}}}{d\left(X_{i}^{(k-1)}, a_{j_{i}}\right)}}
$$

where the superscript $k$ refers to the iteration number within the Weiszfeld iterative procedure; $\left(a_{j_{i}}^{1}, a_{j_{i}}^{2}\right)$ is the location of the set of $n_{i}$ customer points, $j_{i}=1,2, \ldots, n_{i}$; $\left(X_{i}^{1^{(k)}}, X_{i}^{2^{(k)}}\right)$ indicates the new found location of the $i^{\text {th }}$ facility at iteration $k(i=1,2, \ldots, M)$ and $w_{j_{i}}$ designates to all or a fraction of the $j^{\text {th }}$ customer demand that is served by facility $i$.

Figure 2 The main steps of cooper's ATL heuristic

\begin{tabular}{|ll|}
\hline Step 1 & Generate $M$ initial starting locations at random from customers' locations. \\
Step 2 & Find the corresponding allocation by solving the TP. \\
Step 3 & Find the new location using (5). \\
Step 4 & $\begin{array}{l}\text { Solve the TP to obtain the new corresponding allocation based on the new locations } \\
\text { and its cost. }\end{array}$ \\
Step 5 & $\begin{array}{l}\text { Iterate steps } 3 \text { and } 4 \text { until there are no further changes in cost, within some tolerance, } \\
\text { in two successive iterations. }\end{array}$ \\
\hline
\end{tabular}

\subsection{The BCA}

An illustration of the BCA is shown in Figure 3. Firstly, square cells are constructed which will cover all demand points with a side $\delta$ and then empty cells (cells with no customers) are deleted. If the number of non-empty cells is not in the range of a prescribed number of aggregated spatial unit (ASU) then the value of $\delta$ is revised and this process step is repeated. Once the specified number of the non-empty cells, $\rho$, is reached, 
a point or a potential location is chosen randomly from each cell to represent the aggregated point (ASU) within that cell. Finally, to increase the diversity of the solutions, the remaining $(m-\rho)$ ASUs are generated at random. The detailed algorithm of the BCA is given in Figure 4.

Figure 3 The illustration of the BCA (see online version for colours)

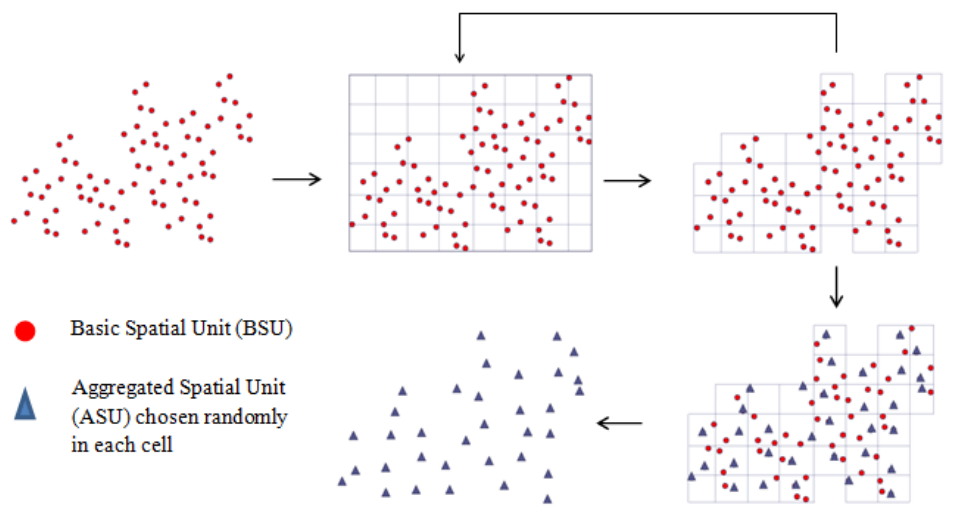

Figure 4 The main step by step of the BCA

\begin{tabular}{|c|c|}
\hline Step 1 & Define the parameters the number of ASUs $(m), \gamma$, and $\lambda$. \\
\hline \multirow[t]{3}{*}{ Step 2} & Initialise the length of the side of the cell $\delta$ as follows: \\
\hline & $\delta=\left(x_{\max }-x_{\min }\right) / \sqrt{m\left(\frac{x_{\max }-x_{\min }}{y_{\max }-y_{\min }}\right)}$ \\
\hline & $\begin{array}{l}\text { where } x_{\max } \text { and } x_{\min } \text { refer to the maximum and the minimum } x \text { coordinates of the } \\
\text { potential points, respectively. } y_{\max } \text { and } y_{\min } \text { are defined similarly. }\end{array}$ \\
\hline Step 2 & Let $\rho$ denote the number of non-empty cells, where $\rho \in[m \gamma(1-\lambda), m \gamma]$. \\
\hline Step 3 & $\begin{array}{l}\text { Construct square cells of length } \delta \text { which cover all demand points where cell } 1 \text { has its } \\
\text { bottom-left corner at }\left(x_{\min }, y_{\min }\right) \text {. If }(\rho \in[m \gamma(1-\lambda), m \gamma] \text { then go to step } 8 \text {. }\end{array}$ \\
\hline Step 4 & $\begin{array}{l}\text { Let } \delta_{L} \text { and } \delta_{U} \text { be lower and upper bounds of the length of the side of the cell. Set } \\
\delta_{\mathrm{U}}=\delta \text { and } \delta_{L}=\delta_{U} / 2 \text {. }\end{array}$ \\
\hline Step 5 & $\begin{array}{l}\text { Construct square cells of length } \delta_{L} \text {. If } \rho<m \gamma(1-\lambda) \text { then set } \delta_{U}=\delta_{L} \text { and } \delta_{L}=\delta_{U} / 2 \text { and } \\
\text { repeat this step, otherwise conduct the bisection method as in step } 6 \text {. }\end{array}$ \\
\hline Step 6 & Calculate $\delta=\left(\delta_{U}+\delta_{L}\right) / 2$ \\
\hline Step 7 & $\begin{array}{l}\text { Construct square cells of length } \delta \text {. If } \rho \geq m \gamma(1-\lambda) \text { and } \rho \leq m \gamma \text { then go to step } 8 \text {, } \\
\text { otherwise, if } \rho<m \gamma(1-\lambda) \text { then } \delta_{U}=\delta \text { else } \delta_{L}=\delta \text { and go back to step } 6 \text {. }\end{array}$ \\
\hline Step 8 & Set count $=0$ \\
\hline Step 9 & Generate randomly $\beta \in(0,1)$ and choose $\tilde{z}$ st $\tilde{z}=F_{(z)}^{-1}(\beta)$ with $F_{(z)}=\sum_{v=1}^{z} P_{v}$. \\
\hline Step 10 & Choose randomly a demand point $k$ in the cell $\tilde{z}$. \\
\hline Step 11 & $\begin{array}{l}\text { Set } X=X \bigcup\{k\} \text {. If }|X|=M \text { stop the search. Otherwise set count }=\text { count }+1 \text { and return } \\
\text { to step } 9 .\end{array}$ \\
\hline
\end{tabular}




\subsection{The proposed VNS algorithm}

In stage 2, the implementation of the metaheuristic VNS is adopted to enhance the solutions obtained from the first stage. The aim is to reduce the risk of being trapped at poor local minima. Figure 5 presents the main steps of the proposed VNS to tackle the CMSWP. Let $c_{\max }$ denote the number of cycles (times) for executing the VNS. In the VNS, the neighbourhood search is conducted by 'shaking' the current solution. In our method, the shaking process is done by swapping a randomly chosen facility with a randomly facility chosen to diversify the search even more. In the local search, the Cooper's ATL heuristic is implemented.

Figure 5 The proposed VNS algorithm to solve the CMSWP

\begin{tabular}{|l}
\hline Step 1 Repeat $c_{\max }$ times the following steps: \\
Step 1.1 Set counter $k=1$ \\
Step 1.2 Shaking \\
Do the following step $k$ times. \\
- Remove randomly 1 facility from $X$ and add 1 facility randomly as well. The new \\
facility is chosen at random from one of customer locations. \\
Step $1.3 \quad$ Local search \\
- Apply local search (Cooper's ATL heuristic) starting from $X$. \\
Step $1.4 \quad$ Move or not \\
If there is an improvement, update the solutions and set $k=1$ else \\
$k=k+1$. \\
Step $1.5 \quad$ If $k \leq k_{\max }$ then return to step 1.2, else go to step 2 \\
Keep the best solution as the final result. \\
Step 2
\end{tabular}

\section{Computational experiments}

To evaluate the performance of the proposed two-stage method, the experiments were conducted using the well-known datasets taken from Brimberg et al. (2000). These datasets were originally used for the case of MSWP. Initially, there were four datasets in Brimberg et al. (2000), which consist of 50, 287, 654, and 1,060 customer points, respectively. In this experiment, we only use three datasets to represent medium and large problems, i.e., 287, 654, and 1,060 customer points.

The number of open facilities $(M)$ is set to be from 5 to 50 with an increment of 5 . Since these datasets were originally used for the uncapacitated cases, hence Zainuddin and Salhi (2007) suggested that the capacity of facility $i\left(b_{i}\right)$ is computed as the average demand of each facility, such that $b_{i}=\left\lceil\sum_{j=1}^{n} w_{j} / M\right\rceil$, where $\lceil x\rceil$ is the smallest integer $\geq x$. This setting is also adopted by Luis et al. $(2009,2011)$. It is also observed that there would be cases where the total capacity of the facilities (i.e., $M b_{i}$ ) would be larger than the total customer demand $\left(\sum_{j=1}^{n} w_{j}\right)$. In this case, such as $M b_{i}>\sum_{j=1}^{n} w_{j}$, a dummy 
customer with a 0 transportation cost and a demand given by $\left(M b_{i}-\sum_{j=1}^{n} w_{j}\right)$ will be introduced. This dummy customer will only contribute at the TP stage and will be ignored during the location and the allocation processes.

The proposed VNS algorithm was implemented in C++ .Net 2012 and run on a PC with an Intel Core i5 CPU @ $3.20 \mathrm{GHz}$ processor, $8.00 \mathrm{~GB}$ of RAM and under Windows 7. Some parameters were used during the execution of the proposed VNS algorithm, i.e., $k_{\max }=M, c_{\max }=M, m=2 * M, \gamma=1-0.1$, and $\lambda=0.05$. The figures of these parameters were found based on our preliminary experiments.

The solutions of the MSWP provided by Brimberg et al. (2000) are used as lower bounds for the CMSWP. The percentage deviation is computed based on these lower bounds as follows:

$$
\operatorname{dev}(\%)=\frac{F_{b e s t}-F_{L B}}{F_{L B}} \times 100
$$

where $F_{\text {best }}$ designates to the best solution obtained by the proposed VNS algorithm and $F_{L B}$ refers to the lower bound or 'best' cost for the MSWP.

Table 1 Comparison for all the results for the 287-customers problem

\begin{tabular}{|c|c|c|c|c|c|c|c|c|c|}
\hline \multirow{2}{*}{$M$} & \multirow{2}{*}{$\begin{array}{l}\text { Lower } \\
\text { bound }\end{array}$} & \multicolumn{2}{|r|}{$Z S$} & \multicolumn{2}{|c|}{$L S N$} & \multicolumn{2}{|c|}{$G L S N$} & \multicolumn{2}{|c|}{$\begin{array}{l}\text { The proposed } \\
\text { VNS algorithm }\end{array}$} \\
\hline & & $\begin{array}{l}\text { Dev. } \\
(\%)\end{array}$ & $\begin{array}{l}C P U \\
(\mathrm{sec})\end{array}$ & $\begin{array}{l}\text { Dev. } \\
(\%)\end{array}$ & $\begin{array}{l}C P U \\
(\mathrm{sec})\end{array}$ & $\begin{array}{l}\text { Dev. } \\
(\%)\end{array}$ & $\begin{array}{l}C P U \\
(\mathrm{sec})\end{array}$ & $\begin{array}{l}\text { Dev. } \\
(\%)\end{array}$ & $\begin{array}{l}C P U \\
(\mathrm{sec})\end{array}$ \\
\hline 5 & $9,715.63$ & 7.90 & 56.57 & 7.92 & 11 & 7.92 & 14 & 7.94 & 110,662 \\
\hline 10 & $6,705.04$ & 25.77 & 539.11 & 25.80 & 17 & 25.80 & 41 & 25.80 & 487,580 \\
\hline 15 & $5,224.70$ & 33.82 & $1,837.17$ & 33.89 & 25 & 33.99 & 118 & 33.87 & $1,250,507$ \\
\hline 20 & $4,148.84$ & 38.99 & $2,532.21$ & 38.48 & 38 & 38.48 & 251 & 38.44 & $1,586,951$ \\
\hline 25 & $3,348.71$ & 44.37 & $3,442.54$ & 44.33 & 64 & 44.31 & 478 & 44.27 & $1,537,624$ \\
\hline 30 & $2,716.91$ & 54.23 & $6,265.81$ & 54.15 & 85 & 54.14 & 792 & 54.10 & $1,657,516$ \\
\hline 35 & $2,238.18$ & 69.27 & $8,310.36$ & 69.24 & 125 & 69.23 & 1,202 & 69.17 & $3,126,617$ \\
\hline 40 & $1,900.84$ & 84.96 & $10,948.99$ & 84.33 & 151 & 84.33 & 1,688 & 84.26 & $2,115,969$ \\
\hline 45 & $1,630.31$ & 94.82 & $12,169.82$ & 94.92 & 219 & 94.82 & 2,530 & 94.89 & $2,731,977$ \\
\hline 50 & $1,402.58$ & 115.94 & $19,814.90$ & 116.06 & 312 & 115.93 & 3,815 & 115.86 & $3,974,198$ \\
\hline \multicolumn{2}{|c|}{ OAV } & 57.01 & $6,601.76$ & 56.91 & 104.70 & 56.89 & $1,092.90$ & 56.86 & \\
\hline
\end{tabular}

To the best of our knowledge, the results of Zainuddin and Salhi (2007), ZS for short, Luis et al. (2009), LSN for short, and Luis et al. (2011), GLSN for short are the only ones available in the literature for direct comparison. The summary results for the three datasets are presented in Tables 1 to 3, respectively. For completeness, the overall average (OAV) for each dataset is also given. The ital numbers in Tables 1 to 3 refer to the best new found solutions. Based on our results, the proposed method provides encouraging solutions when compared to the results from the literature. Our results are superior from the results given by Zainuddin and Salhi (2007) and Luis et al. (2009) and very competitive compared to Luis et al. (2011). For instance, in the case of the 287-customers problem, the proposed method found six new best solutions and the OAV 
outperform the results of Zainuddin and Salhi (2007) and Luis et al. $(2009,2011)$ by $0.26 \%, 0.09 \%$, and $0.05 \%$. In the case of the 654 -customers problem, the proposed method found one new best solutions. In the case of $n=1,060$ customers, the proposed method gives better results by $1 \%$ and $0.50 \%$ from Zainuddin and Salhi (2007) and Luis et al. (2009) respectively and the proposed method found five new best solutions out of ten. However, based on the OAV, the proposed method still provides favourable results compared to the GLSN of Luis et al. (2011).

To diversify the search, our proposed two-stage method explores different neighbourhoods by swapping potential facility locations. However, as we introduce a multi-start approach within the search, the proposed method requires more computing time.

Table 2 Comparison for all the results for the 654-customers problem

\begin{tabular}{|c|c|c|c|c|c|c|c|c|c|}
\hline \multirow{2}{*}{$M$} & \multirow{2}{*}{$\begin{array}{l}\text { Lower } \\
\text { bound }\end{array}$} & \multicolumn{2}{|r|}{$Z S$} & \multicolumn{2}{|c|}{$L S N$} & \multicolumn{2}{|c|}{$G L S N$} & \multicolumn{2}{|c|}{$\begin{array}{c}\text { The proposed VNS } \\
\text { algorithm }\end{array}$} \\
\hline & & $\begin{array}{l}\text { Dev. } \\
(\%)\end{array}$ & $\begin{array}{l}C P U \\
(\mathrm{sec})\end{array}$ & $\begin{array}{l}\text { Dev. } \\
\text { (\%) }\end{array}$ & $\begin{array}{l}C P U \\
(\mathrm{sec})\end{array}$ & $\begin{array}{l}\text { Dev. } \\
(\%)\end{array}$ & $\begin{array}{l}C P U \\
(\mathrm{sec})\end{array}$ & $\begin{array}{l}\text { Dev. } \\
(\%)\end{array}$ & $\begin{array}{l}C P U \\
(\mathrm{sec})\end{array}$ \\
\hline 5 & $9,715.63$ & 54.00 & 62.67 & 54.00 & 18 & 54.00 & 25 & 54.00 & 215,555 \\
\hline 10 & $6,705.04$ & 42.81 & 136.94 & 42.81 & 31 & 42.81 & 81 & 42.81 & 459,415 \\
\hline 15 & $5,224.70$ & 67.69 & 852.92 & 67.69 & 64 & 67.69 & 355 & 67.69 & 859,849 \\
\hline 20 & $4,148.84$ & 69.37 & $1,086.59$ & 69.36 & 107 & 69.36 & 726 & 69.36 & $1,788,603$ \\
\hline 25 & $3,348.71$ & 47.52 & $3,842.79$ & 47.52 & 180 & 47.51 & 1,331 & 47.52 & $7,523,410$ \\
\hline 30 & $2,716.91$ & 86.77 & 852.18 & 76.33 & 247 & 76.33 & 2,199 & 76.33 & $9,113,601$ \\
\hline 35 & $2,238.18$ & 78.13 & $5,772.53$ & 78.13 & 354 & 78.13 & 3,455 & 78.13 & $12,558,625$ \\
\hline 40 & $1,900.84$ & 44.25 & $4,740.56$ & 43.85 & 517 & 43.84 & 5,112 & 43.84 & $13,148,668$ \\
\hline 45 & $1,630.31$ & 59.23 & $5,112.34$ & 55.28 & 638 & 55.26 & 7,265 & 55.26 & $18,756,620$ \\
\hline 50 & $1,402.58$ & 41.96 & $20,333.38$ & 29.13 & 807 & 29.13 & 9,884 & 29.12 & $15,897,503$ \\
\hline \multicolumn{2}{|c|}{ OAV } & 59.17 & $4,279.29$ & 56.41 & 296.30 & 56.40 & $3,043.30$ & 56.40 & \\
\hline
\end{tabular}

Table 3 Comparison for all the results for the 1,060-customers problem

\begin{tabular}{|c|c|c|c|c|c|c|c|c|c|}
\hline \multirow{2}{*}{$M$} & \multirow{2}{*}{$\begin{array}{l}\text { Lower } \\
\text { bound }\end{array}$} & \multicolumn{2}{|r|}{$Z S$} & \multicolumn{2}{|c|}{$L S N$} & \multicolumn{2}{|c|}{$G L S N$} & \multicolumn{2}{|c|}{$\begin{array}{c}\text { The proposed VNS } \\
\text { algorithm }\end{array}$} \\
\hline & & $\begin{array}{l}\text { Dev. } \\
(\%)\end{array}$ & $\begin{array}{l}C P U \\
(\mathrm{sec})\end{array}$ & $\begin{array}{l}\text { Dev. } \\
(\%)\end{array}$ & $\begin{array}{l}C P U \\
(\mathrm{sec})\end{array}$ & $\begin{array}{l}\text { Dev. } \\
(\%)\end{array}$ & $\begin{array}{l}C P U \\
(\mathrm{sec})\end{array}$ & $\begin{array}{l}\text { Dev. } \\
(\%)\end{array}$ & $\begin{array}{l}C P U \\
(\mathrm{sec})\end{array}$ \\
\hline 5 & $1,851,879.88$ & 1.06 & 370.85 & 1.06 & 60 & 1.06 & 72 & 1.06 & 206,564 \\
\hline 10 & $1,249,564.75$ & 3.11 & $1,167.35$ & 3.11 & 101 & 3.11 & 264 & 3.11 & $1,330,936$ \\
\hline 15 & $980,132.13$ & 1.63 & $4,063.94$ & 1.63 & 255 & 1.63 & 845 & 1.63 & $5,424,609$ \\
\hline 20 & $828,802.00$ & 3.42 & $5,116.90$ & 3.35 & 436 & 3.35 & 1833 & 3.33 & $12,549,620$ \\
\hline 25 & $722,061.19$ & 3.87 & $30,551.64$ & 3.91 & 662 & 3.85 & 3261 & 3.87 & $14,588,140$ \\
\hline 30 & $638,263.00$ & 3.92 & $45,191.83$ & 3.95 & 1002 & 3.92 & 5311 & 3.93 & $17,450,855$ \\
\hline 35 & $577,526.63$ & 3.35 & $21,713.60$ & 3.36 & 1365 & 3.31 & 8253 & 3.31 & $20,181,102$ \\
\hline 40 & $529,866.19$ & 6.02 & $47,253.61$ & 6.16 & 1700 & 5.99 & 12754 & 6.04 & $24,628,143$ \\
\hline 45 & $489,650.00$ & 7.83 & $12,0072.31$ & 7.88 & 2204 & 7.74 & 17125 & 8.01 & $29,593,225$ \\
\hline 50 & $453,164.00$ & 5.87 & $13,0753.06$ & 5.50 & 2797 & 5.29 & 23854 & 5.40 & $37,597,025$ \\
\hline \multicolumn{2}{|c|}{ OAV } & 4.01 & $40,625.51$ & 3.99 & $1,058.00$ & 3.92 & $7,357.20$ & 3.97 & $16,355,021.90$ \\
\hline
\end{tabular}




\section{Conclusions}

This study has put forward the two-stage method to tackle the capacitated multisource Weber problem. In the first stage, initial facility configurations are generated using the BCA. In the second stage, the improvement is made through the implementation of the VNS. Our results found seven new best solutions out of 30 solutions. This has shown that our proposed two-stage method produce competitive results when compared to the existing results in the literature.

Potential future investigations based on the current study may include implementations on other metaheuristic techniques such as large neighbourhood search or evolutionary algorithms, see Gendreau and Potvin (2010) for a comprehensive review on metaheuristics. The inclusion of set up cost for constructing facilities in the objective function can be one of the new research areas.

\section{References}

Akyüz, M.H., Altınel, I.K. and Öncan, T. (2014) 'Location and allocation based branch and bound algorithms for the capacitated multi-facility Weber problem', Annals of Operations Research, Vol. 222, No. 1, pp.45-71.

Aras, N., Altınel, I.K. and Orbay, M. (2007a) 'New heuristic methods for the capacitated multi-facility Weber problem', Naval Research Logistics, Vol. 54, No. 5, pp.21-32.

Aras, N., Orbay, M. and Altinel, I.K. (2008) 'Efficient heuristics for the rectilinear distance capacitated multi-facility Weber problem', Journal of the Operational Research Society, Vol. 59, No. 1, pp.64-79.

Aras, N., Yumusak, S. and Altınel, I.K. (2007b) 'Solving the capacitated multi-facility Weber problem by simulated annealing, threshold accepting and genetic algorithms', in Doerner, K.F. et al. (Eds.): Metaheuristics: Progress in Complex Systems Optimization, pp.91-112, Springer.

Brimberg, J. and Mladenović, N. (1996) 'Variable neighbourhood algorithm for solving the continuous location-allocation problem', Studies in Locational Analysis, Vol. 10, No. 1, pp.1-12.

Brimberg, J., Drezner, Z., Mladenović, N. and Salhi, S. (2014) 'A new local search for continuous location problems', European Journal of Operational Research, Vol. 232, No. 2, pp.256-265.

Brimberg, J., Hansen, P., Mladenović, N. and Salhi, S. (2008) 'A survey of solution methods for the continuous location-allocation problem', International Journal of Operations Research, Vol. 5, No. 1, pp.1-12.

Brimberg, J., Hansen, P., Mladenović, N., and Taillard, E. D. (2000) 'Improvements and comparison of heuristics for solving the uncapacitated multisource Weber problem', Operations Research, Vol. 48, No. 3, pp.444-460.

Cooper, L. (1964) 'Heuristic methods for location-allocation problems', SIAM Review, Vol. 6, No. 1, pp.37-53.

Cooper, L. (1972) 'The transportation-location problem', Operations Research, Vol. 20, No. 1, pp.94-108.

Drezner, Z. (2015) 'The fortified Weiszfeld algorithm for solving the Weber problem', IMA Journal of Management Mathematics, Vol. 26, No. 1, pp.1-9.

Farham, M.S., Süral, H. and Cem Iyigun, C. (2015) 'The Weber problem in congested regions with entry and exit points', Computers and Operations Research, Vol. 62, No. 1, pp.177-183. 
Fernandes, I.F., Aloise, D., Aloise, D.J., Hansen, P. and Liberti, L. (2014) 'On the Weber facility location problem with limited distances and side constraints', Optimization Letters, Vol. 8, No. 2, pp.407-424.

Gamal, M.D.H. and Salhi, S. (2003) 'A cellular heuristic for the multisource Weber problem', Computers and Operations Research, Vol. 30, No. 11, pp.1609-1624.

Gendreau, M. and Potvin, J-Y. (2010) Handbook of Metaheuristics of International Series in Operations Research and Management Science, Springer, Boston.

Hansen, P. and Mladenović, N. (1997) 'Variable neighbourhood search for the p-median', Location Science, Vol. 5, No. 4, pp.207-225.

Hansen, P. and Mladenović, N. (2001) 'Variable neighborhood search: principles and applications', European Journal of Operational Research, Vol. 130, No. 3, pp.449-467.

Hansen, P., Mladenović, N. and Moreno Perez, J.A. (2010) 'Variable neighborhood search: methods and applications', Annals of Operations Research, Vol. 175, No. 4, pp.367-407.

Hansen, P., Mladenović, N. and Taillard, E.D. (1998) 'Heuristic of the multisource Weber problem as a p-median problem', Operation Research Letters, Vol. 22, Nos. 2-3, pp.55-62.

Hosseininezhad, S.J., Salhi, S. and Jabalameli, M.S. (2015) 'A cross entropy-based heuristic for the capacitated multi-source Weber problem with facility fixed cost', Computers and Industrial Engineering, Vol. 83, No. 1, pp.151-158.

Irawan, C.A. and Salhi, S. (2015) 'Solving large p-median problems by a multistage hybrid approach using demand points aggregation and variable neighbourhood search', Journal of Global Optimization, Vol. 63, No. 3, pp.537-554.

Jamalian, A. and Salahi, M. (2014) 'Robust solutions to multi-facility Weber location problem under interval and ellipsoidal uncertainty', Applied Mathematics and Computation, Vol. 242, No. 1, pp.179-186.

Luis, M., Salhi, S. and Nagy, G. (2009) 'Region-rejection based heuristics for the capacitated multi-source Weber problem', Computers and Operations Research, Vol. 36, No. 6, pp.2007-2017.

Luis, M., Salhi, S. and Nagy, G. (2011) 'A guided reactive GRASP for the capacitated multi-source Weber problem', Computers and Operations Research, Vol. 38, No. 7, pp.1014-1024.

Mohammadi, N., Malek, M.R. and Alesheikh, A.A. (2010) 'A new GA based solution for capacitated multi source Weber problem', International Journal of Computational Intelligence Systems, Vol. 3, pp.514-521.

Sherali, H.D. and Shetty, C.M. (1977) 'The rectilinear distance location-allocation problem', AIIE Transaction, Vol. 9, No. 2, pp.136-143.

Sherali, H.D. and Tuncbilek, C.H. (1992) 'A squared-Euclidean distance location-allocation problem', Naval Research Logistics, Vol. 39, No. 4, pp.447-469.

Sherali, H.D., Al-Loughani, I. and Subramanian, S. (2002) 'Global optimization procedures for the capacitated Euclidean and $\ell_{P}$ distance multifacility location-allocation problem', Operations Research, Vol. 50, No. 3, pp.433-448.

Sherali, H.D., Ramachandran, S. and Kim, S. (1994) 'A localization and reformulation discrete programming approach for the rectilinear distance location-allocation problem', Discrete Applied Mathematics, Vol. 49, Nos. 1-3, pp.357-378.

Uno, T., Kato, K. and Katagiri, H. (2015) 'Fuzzy random weighted Weber problems in facility location', Procedia Computer Science, Vol. 60, No. 1, pp.936-943.

Zainuddin, M.Z. and Salhi, S. (2007) 'A perturbation-based heuristic for the capacitated multisource Weber problem', European Journal of Operational Research, Vol. 179, No. 3, pp.1194-1207. 